\title{
Development of Aerosol Models for Radiative Flux Calculations at ARM Sites
}

John A. Ogren and Ellsworth G. Dutton,

NOAA Earth Sciences Research Laboratory, Boulder, Colorado

The direct radiative forcing (DRF) of aerosols, the change in net radiative flux due to aerosols in noncloudy conditions, is an essential quantity for understanding the human impact on climate change. Our work has addressed several key issues that determine the accuracy, and identify the uncertainty, with which aerosol DRF can be modeled. These issues include the accuracy of several radiative transfer models when compared to measurements and to each other in a highly controlled closure study (Michalsky et al., 2006) using data from the ARM 2003 Aerosol IOP. The primary focus of our work has been to determine an accurate approach to assigning aerosol properties appropriate for modeling over averaged periods of time and space that represent the observed regional variability of these properties (McComiskey et al., in prep.; Payton et al., 2004). We have also undertaken a comprehensive analysis of the aerosol properties that contribute most to uncertainty in modeling aerosol DRF, and under what conditions they contribute the most uncertainty (McComiskey et al., 2006). Quantification of these issues enables the community to better state accuracies of radiative forcing calculations and to concentrate efforts in areas that will decrease uncertainties in these calculations in the future.

The accuracy with which aerosol DRF is known is demonstrated through closure studies, which involve the comparison of radiation measurements and the same quantity calculated by a radiative transfer model. Results depend on how well a model simulates physical processes and the accuracy of the aerosol and radiation measurements. We have shown that where instantaneous, collocated measurements of aerosol properties and surface radiation exist, closure of $<1 \%$ for the direct radiation component and $<2 \%$ for the diffuse radiation component has been achieved with several different radiative transfer models (Michalsky et al. 2006). These results provide confidence in model physics and measurement techniques, but the temporal and spatial scales of interest to climate change studies require some amount of averaging of instantaneous measurements. Calculating an accurate aerosol DRF for pertinent time scales depends on whether the model inputs are truly representative of the region or temporal entity for which they are intended.

Aerosol characteristics can change on timescales of less than 10 hours and may be highly variable over distances as short as $\sim 20 \mathrm{~km}$, depending on meteorology, sources, transformations, and sinks. Such geographic variability causes significant diversity in local and regional impacts that can affect heating rates and atmospheric circulation around the globe, However, these impacts are difficult to predict with much certainty because the regional characteristics of aerosol are not well defined. In order to address the error in radiative transfer modeling of aerosol associated with spatial and temporal variability, we have developed an approach to defining aerosol characteristics on a regional scale for two DOE ARM sites. These two sites, the Southern Great Plains (SGP) and North Slope of Alaska (NSA) have existing records from long-term, comprehensive monitoring of aerosol properties in different environments. The SGP site, located in central Oklahoma, began measurement of aerosol properties representing US continental conditions in 1996. At Barrow, Alaska adjacent to ARM's North Slope of Alaska (NSA) site, over 20 years of data are available from NOAA's Baseline Observatory, representing Arctic marine conditions. Data from these two sites are quantitatively comparable, as the measurements of aerosol optical properties, chemistry, and local meteorology have been made with the same or very similar instruments through the years.

Aerosol types have been defined using a k-means clustering algorithm to identify patterns among chemistry, meteorology and aerosol optical properties inherent in the long-term surface data. Variables included in the analysis are those that are directly measured, rather than derived and were found to most strongly differentiate aerosol type. These include chemical composition (cation and anion concentrations); absorption, scattering and backscattering coefficients; aerosol number concentration; and dew point 
temperature. The dependence of aerosol light scattering with changes in relative humidity, $f(\mathrm{RH})$, is included in the SGP analysis, but $f(\mathrm{RH})$ measurements are not made at NSA. Daily averages of these properties are used in the clustering algorithm for SGP. The averaging time for chemical composition at NSA ranges from one day in the winter to up to four days in the summer and the cases included in the analysis reflect this variable averaging period. Each variable for each case must have a valid value for the clustering to be successful so any case with missing data must be thrown out, resulting in a reduced number of cases for the time periods of data collection at each site given below. Once the clustering results were obtained, a name was subjectively assigned to each aerosol type according to their dominant chemical characteristics and mean optical properties.

Four dominant aerosol types were found to exist at SGP: (1) background, (2) industrial, (3) burning, and (4) dust. Of 702 cases between 2000 and 2005, their percent occurrences and median optical properties for the mass extinction efficiency (Qext $(\mathrm{m} 2 / \mathrm{g})$ ), single scattering albedo (SSA), and asymmetry factor (ASM) are shown in the table below. While background aerosol is seen at SGP throughout the year, the industrial aerosol shows a seasonal cycle throughout the spring, summer, and fall, likely due to prevalent meteorological conditions. Burning and dust events are rare and episodic, mostly in spring and summer. Five-day back trajectories were analyzed for each aerosol type and show that particular aerosol types do not appear to originate from specific source regions.

Four dominant aerosol types were also found to exist at NSA: (1) background, (2) haze, (3) sea salt, and (4) a dust and sea salt mixture. Based on 360 cases between 1997 and 2003, their percent occurrences and optical properties are also shown in the table below. Background and haze aerosol are dominant in the wintertime and sea salt is dominant in the summertime after sea ice retreat. A few possible dust events are seen during the winter although the signal is not clearly for dust. Trajectory analysis shows that background conditions exist primarily when air masses are from the pole and haze conditions exist when air masses originate from the North American continent. The few possible dust events show a trajectory from the direction of east Asia.

$\begin{array}{lllll} & \text { \% occurrence } & \text { Qext. }(\mathrm{m} 2 / \mathrm{g}) & \text { SSA } & \text { ASM } \\ \text { SGP } & & & & \\ \text { Background } & 85 \% & 3.9 & 0.93 & 0.60 \\ \text { Industrial } & 12 \% & 4.6 & 0.95 & 0.65 \\ \text { Burning } & 2 \% & 4.5 & 0.88 & 0.55 \\ \text { Dust } & 1 \% & 2.1 & 0.92 & 0.59 \\ \text { NSA } & & & & \\ \text { Background } & 48 \% & 3.3 & 0.94 & 0.66 \\ \text { Haze } & 42 \% & 2.2 & 0.97 & 0.67 \\ \text { Sea salt } & 9 \% & 3.0 & 0.96 & 0.60 \\ \text { Sea salt/dust } & 1 \% & 2.2 & 0.97 & 0.69\end{array}$

When modeling aerosol on regional scales, values for these optical properties are often specified based on observations at one or a few points in time within that region or even on an assumed global average value. This long-term analysis of aerosol properties at key ARM site will provide better estimates of these properties for modeling at these sites and a guideline for understanding the uncertainties incurred in averaging properties over space and time.

The uncertainty in calculated aerosol DRF is a function of the uncertainty in the aerosol properties that are specified in the calculations. We have examined the uncertainty of DRF due to uncertainty in the quantities on which it depends: aerosol optical properties, i.e., single scattering albedo and asymmetry parameter, and situational variables, i.e., solar geometry and surface albedo, and the wavelength dependencies of these quantities. The DRF for net irradiance at the top of the atmosphere (TOA) and surface is computed for three base cases representing three different radiative environments with distinct 
aerosol assemblages: (1) Tropical Western Pacific, (2) Southern Great Plains, and (3) North Slope of Alaska. For each case, the DRF is presented for three scenarios (1) integrated over the shortwave and averaged over solar zenith angle for the Spring Equinox, (2) at $0.55 \mu \mathrm{m}$ and averaged over solar zenith angle for the Spring Equinox, and (3) integrated over the shortwave at $30^{\circ}, 45^{\circ}$, and $70^{\circ}$ solar zenith angle. While the magnitude of the uncertainty in DRF relates directly to the magnitude of the measurement uncertainty in aerosol and situational properties, the absolute and relative effects of the several uncertainties are dependent on the relationships between aerosol optical properties and environmental variables and can vary greatly.

We have defined these uncertainties for the conditions represented by each of the three ARM sites by calculating a sensitivity value of the DRF to each of the above quantities of interest. The sensitivity is defined as the derivative of the DRF with respect to the property of interest, multiplied by the measurement uncertainty of that property. In general, calculated DRF shows the greatest sensitivity to single scattering albedo for the three scenarios, followed by asymmetry parameter, surface albedo, and optical depth. While the measurement uncertainty of the asymmetry parameter is greater that that for the single scattering albedo, the latter has a larger impact on the calculated DRF and therefore a greater sensitivity in most cases. An example of an exception when the asymmetry parameter sensitivity is higher than for the single scattering albedo is the top of the atmosphere DRF for TWP and SGP conditions where the surface albedo is low and the change in directional scattering more significantly impacts the change in flux at the top of the atmosphere that changes in single scattering albedo. While optical depth is know to be the most important factor in determining the magnitude of aerosol radiative forcing, the current measurement uncertainties are low and do not contribute as must to the uncertainties in calculated DRF. This information can guide efforts in improvement of measurement uncertainties and the required accuracy with which aerosol properties must be specified for radiative forcing calculations to provide confidence in aerosol radiative forcing estimates.

\section{References}

McComiskey, A., S. E. Schwartz, J. A. Ogren, J. J. Michalsky, E. R. Lewis, P. Ricchiazzi, Direct Aerosol Forcing: Sensitivity to Uncertainty in Measurements of Aerosol Optical and Situational Properties, The Sixteenth ARM Science Team Meeting Proceedings, Albuquerque, Florida, March 27-30, 2006.

McComiskey, A., J. A. Ogren, E. G. Dutton, J. M. Harris, and P., K. Quinn. An Empirical Approach to Regional Aerosol Model Development for Radiative Transfer Calculations. In preparation.

Michalsky, J. J., G. P. Anderson, J. Barnard, J. Delamere, C. Gueymard, S. Kato, P. Kiedron, A. McComiskey, P. Ricchiazzi. 2006. Shortwave radiative closure studies for clear skies during the Atmospheric Radiation Measurement 2003 Aerosol Intensive Observation Period. J. Geophys. Res., Vol. 111, No. D14, D14S90, 10.1029/2005JD006341.

Payton, A., J.A. Ogren, E. Dutton, P. Quinn, J. Harris, E. Andrews. 2004. An Empirical Approach to Aerosol Model Development for Radiative Transfer Calculations. The Fourteenth ARM Science Team Meeting Proceedings, Albuquerque, New Mexico, March 22 - 26, 2004. 